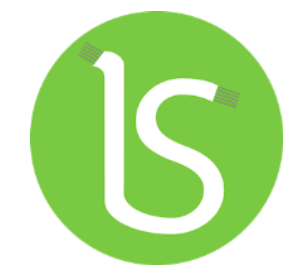

\title{
Mercados diferenciados en organizaciones cafetaleras del centro de Veracruz, México
}

\author{
Liliana Ramos Rivera a \\ Martha Elena Nava Tabalada b \\ Esteban Escamilla Prado c
}

\author{
CÓMO CITAR \\ HOW TO CITE: \\ Ramos-Rivera, L., Nava- \\ Tablada, M. E., y Escamilla- \\ Prado, E. (202I). Mercados \\ diferenciados en organizaciones \\ cafetaleras del centro de \\ Veracruz, México. \\ Interconectando Saberes, (12), \\ I77-P. \\ https://doi.org/ 10.25009/is.v0il 2 \\ .2716
}

Recibido: 16 de junio de 2021

Aceptado: 5 de julio de 2021

Publicado: 20 de julio de 2021

Abstract - Mexico is among the top ten coffee producers and Veracruz ranks second nationally. Since the end of the 20th century there has been a crisis derived from the liberation of the international market, overproduction, and low prices. In Mexico, it translates into privatization policies that have a negative impact on the coffee growing regions. Some coffee growers have been able to keep venturing into differentiated markets that give a premium to the product. The objective was to identify the problems and challenges faced by eight organizations to commercialize coffee in differentiated markets. A semi-structured interview was applied to the leaders and the data were analyzed qualitatively. The main problems are reduced niches in specialty markets, poor marketing, high export costs and low domestic consumption. The challenges: increase productivity and quality, improve marketing strategies, and promote national consumption.

Keywords - Coffee production, Marketing, Organization, Alternative Markets, Veracruz.

\footnotetext{
a Colegio de Veracruz, México. E-mail: lilianaramosrivera@gmail.com

b Universidad Veracruzana, México. E-mail: menavata@yahoo.com.mx

(iD) 0000-0002-2674-3502

c Universidad Autónoma Chapingo. Huatusco, Veracruz, México. E-mail: espreschoca@yahoo.com.mx 


\section{INTRODUCCIÓN}

El café es una de las bebidas que más se consume en el mundo y México se ubica como el noveno productor de café verde y el primer productor mundial de café orgánico, exportándolo a Estados Unidos y Europa preferentemente. De los trece estados cafetaleros en el país, los principales son Chiapas, Veracruz, Oaxaca y Puebla. Además, el café es el sexto producto agrícola de mayor exportación, del cual dependen más de tres millones de mexicanos involucrados en toda la cadena de valor (CEDRSSA, 2018).

A raíz de la liberación del mercado de café internacional y la sobreproducción mundial, los precios internacionales disminuyeron considerablemente. En México también se implementaron políticas de privatización, a raíz de ello en 1989 desaparece el Instituto Mexicano del Café (INMECAFÉ), instancia que acopiaba y comercializaba el producto y dictaba las políticas cafetaleras, dejando un gran vacío en la cadena de valor, situación que se ha prolongado hasta la actualidad (Hernández-Martínez y Córdova, 20l I).

Al no ser rentable la producción y venta del café, por los bajos precios, los campesinos han combinado este cultivo con otras actividades productivas, como el aprovechamiento de la madera, ganadería y monocultivos como la caña de azúcar o empleándose en la industria de la construcción en las ciudades (Manson et al., 2008). Otros factores han influido en el declive del sector cafetalero, tales como la presencia de plagas y enfermedades, entre ellas la roya que generó el cambio de variedades de arábica a otras que son más resistentes (como la robusta) pero de menor calidad. Igualmente, la roya ocasionó la quema de gran cantidad de plantas, cuya resiembra implicaría al menos 3 años de espera para volver a cosechar (Batista, 2018).

Por otro lado, el cafetal de sombra es importante como recurso ambiental y para el sustento familiar, porque al igual que la milpa, es una forma de producción campesina para autoconsumo, por tratarse de un policultivo, donde se aprovechan otros productos como cítricos, plátanos, hongos, flores y hierbas utilizadas como alimentos, además de sustentar una gran biodiversidad vegetal y animal; es decir, es un bosque comestible (Sosa, 2013). Además de lo señalado, la problemática del café se agrava por el abandono que experimenta la actividad agrícola en general, dado que a las nuevas generaciones ya no les interesa continuar cultivando, por lo que emigran a las ciudades y por consiguiente se pierden saberes productivos locales que se heredan entre generaciones, es decir, los jóvenes no desean continuar las labores productivas en las tierras de sus padres y no aprenden todos los conocimientos acumulados por éstos (Nava-Tablada, 2012).

Con la disminución del cafetal de sombra, se amenaza también al bosque mesófilo de montaña que alberga una gran biodiversidad, dado que el café de altura se produce en este tipo de ecosistema, por lo que es de significativa importancia la conservación de este patrimonio biocultural (Williams-Linera, 2015).

A pesar de la problemática que enfrentan los cafeticultores, muchos de ellos han podido salir adelante y permanecer produciendo, sobre todo al incursionar en la comercialización en mercados diferenciados que otorgan un sobreprecio al grano por su ventaja competitiva (alguna característica que lo distingue del resto de la producción del café) siendo los tres principales mercados: los diferenciados por calidad (gourmet, denominación de origen) las certificaciones 
verdes (orgánico, sustentable, C4, Rainforest Alliance) y Comercio Justo, que implica aspectos organizativos, equidad, justicia social e incluye también aspectos económicos y ambientales (Nava-Tablada, 20I2).

Veracruz es el segundo estado productor de café en México; sobresalen 10 regiones cafetaleras: en la zona norte se ubica Huayacocotla y Papantla; en el sur, Tezonapa y Los Tuxtlas, mientras en el centro se encuentra Zongolica, Córdoba, Misantla, Coatepec, Atzalan y Huatusco (Hermida, 20I8). La zona centro, aporta el $80 \%$ de la producción total estatal de café (Castillo, 2019) y es reconocida en el mercado internacional por su calidad. El ecosistema predominante en dicha zona es el bosque mesófilo de montaña, conocido como bosque de niebla, el cual cuenta con gran biodiversidad y especies de flora y fauna únicas (Manson, 20I3).

Los productores veracruzanos que han incursionado con éxito en la comercialización del café en mercados diferenciados se concentran en la región montañosa central del estado, donde existen varias organizaciones y empresas que venden su producción en ellos, sin embargo, existen pocas investigaciones que aborden la problemática que enfrentan los productores en estos mercados alternativos (Nava-Tablada, 2016).

En este contexto, el objetivo de la presente investigación fue identificar los principales problemas y retos que enfrentan ocho organizaciones y empresas del centro de Veracruz para comercializar café en mercados diferenciados. Para cumplir el objetivo se aplicó una entrevista semiestructurada a los dirigentes y los datos se analizaron cualitativamente.

\section{RESULTADOS}

Las organizaciones y empresas incluidas en la investigación fueron: Catuai Amarillo Sociedad de Solidaridad Social; Unión de Cafetaleros Kolping de las Altas Montañas (UCKAM); Coordinadora de Productores de la Zona Centro del Estado de Veracruz; Consejo Regional de Café de Coatepec (CORECAFECO); Ensambles de Cafés Mexicanos; Vinculación y Desarrollo Agroecológico en Café A.C (VIDA); Productores Sustentables de Ocozaca y Unión Regional de Pequeños Productores de Café de la Región de Huatusco. A continuación, se describen algunas de las características más relevantes de cada una.

I) Catuai Amarillo es una organización integrada por productores de café, propietarios y ejidatarios del municipio de Chocamán, producen café arábiga de calidad y estrictamente de altura que venden en el mercado de café orgánico y Comercio Justo.

2) La UCKAM se encuentra en el municipio de Tepatlaxco y está conformada por pequeños productores que cultivan y procesan café de alta calidad que además promueve el cuidado del medio ambiente.

3) La Coordinadora de Productores de la Zona Centro del Estado de Veracruz es una sociedad cooperativa de productores de café que se localiza en el municipio de Huatusco y ganó en 2015 el primer lugar en el certamen Taza de Excelencia, lo cual corrobora los altos estándares de calidad de su café.

4) EI CORECAFECO busca el desarrollo competitivo a través de café diferenciado de excelente calidad y apoya a los pequeños productores de Coatepec, municipio en el cual 
se localiza; promueve la comercialización de café a granel, tostado y molido en presentaciones individuales.

5) Ensambles de Cafés Mexicanos, se ubica en el municipio de Coatepec y es una red de procesadores, exportadores, productores, importadores, transportistas y tostadores que buscan hacer frente a los actuales retos de la cafeticultura y la industria del café; maneja café de comercio justo, agroecológicos, biodinámicos y orgánicos.

6) VIDA es una asociación civil que tiene como antecedentes largos procesos organizativos y de desarrollo en la región centro de Veracruz; se ubica en el municipio de Ixhuatlán del Café; sus miembros transitaron de la producción de café convencional a una producción orgánica, además de desarrollar otras actividades como el rescate de la medicina tradicional y la diversificación agroecológica en los cafetales.

7) Productores Sustentables de Ocozaca se localiza en el municipio de Ixhuatlán del Café; busca fortalecer las organizaciones de productores otorgándoles capacitación, transferencia de conocimientos y competencias, compartiendo experiencias y técnicas. Comercializa en el mercado orgánico y administra un beneficio seco para todas las organizaciones que le proveen el grano.

8) La Unión Regional de Pequeños Productores de Café de la Región de Huatusco, es una organización de solidaridad social, cuyos miembros decidieron adoptar el cultivo de café orgánico, lo que ha hecho que practiquen una agricultura sustentable, que además busca mejorar la calidad de vida de las familias que dependen del café.

Todas las empresas y organizaciones estudiadas se ubican en la franja cafetalera del centro del estado de Veracruz, que comprende entre los 700 y los 1400 msnm, lo que garantiza que existen las condiciones agroclimáticas óptimas para el cultivo y por tanto se produce grano de calidad (Hermida, 2018).

En cuanto al tipo de café que cultivan o acopian, Catuai Amarillo, la Coordinadora de Productores de la Zona Centro del Estado de Veracruz, VIDA, Productores Sustentables de Ocozaca y UCKAM trabajan con café orgánico certificado, el cual se caracteriza por no usar agroquímicos (fertilizantes, plaguicidas, herbicidas), ya que se rigen por normas internacionales de producción e industrialización que son verificadas por un sistema de certificación. CORECAFECO acopia café diferenciado por calidad, pero cultivado mediante el sistema convencional, que utiliza fertilizantes, pesticidas, herbicidas, fungicidas e insecticidas, que pueden afectar la salud de productores y consumidores. Mientras que la Unión Regional de Pequeños Productores de Café de la Región de Huatusco y Ensambles de Cafés Mexicanos, producen tanto café orgánico como convencional.

Para poder acceder a la comercialización del café en la empresas y organizaciones analizadas, algunas solicitan a los asociados contar con certificación. Sin embargo, UCKAM, Ensambles de Cafés Mexicanos y CORECAFECO no piden ninguna certificación. Por su parte, la Coordinadora de Productores de la Zona Centro del Estado de Veracruz y la Unión Regional de Pequeños Productores de Café de la Región de Huatusco, los Productores Sustentables de Ocozaca, VIDA y Catuai Amarillo requieren la certificación 
orgánica de Fairtrade Labelling Organizations (FLO) y/o CERTIMEX. Catuai además solicita el Distintivo Nacional Orgánico de la Secretaría de Agricultura, Ganadería, Desarrollo Rural, Pesca y Alimentación (SAGARPA) y VIDA las certificaciones NOP-USDA de Estados Unidos y DAkkS que es el organismo de acreditación en Alemania.

Respecto a quiénes financian la cadena del café desde la producción hasta la comercialización, las ocho empresas se consideran autosuficientes, sin embargo, Catuai Amarillo, CORECAFECO y la Coordinadora de Productores de la Zona Centro del Estado de Veracruz, reciben subsidios económicos de programas gubernamentales, mientras que los Productores Sustentables de Ocozaca tienen apoyos de organizaciones religiosas y UCKAM de Kolping Internacional.

A excepción de UCKAM, todas las organizaciones y empresas estudiadas cuentan con una marca sombrilla, es decir, "una marca (que) protege a un producto o líneas de productos bajo su halo de notoriedad o prestigio” (Botey, 1990, p.93), la cual tiene como objetivo "reducir los costes y los riesgos de introducir nuevos productos en el mercado, aprovechando un activo tan importante como es el disponer de una marca con buena reputación, consolidada y fiable. Eso facilita la aceptación del consumidor a los nuevos productos y además minimiza enormemente la inversión necesaria para introducir una nueva marca en el mercado" (Ditsmarketing, 2016, p.l).

En cuanto a qué factores han facilitado la comercialización del café en los mercados diferenciados, Catuai Amarillo mencionó que han sido la organización de los productores, la certificación orgánica y de comercio justo, la excelente calidad en grano y taza, la capacitación de los socios y las alianzas estratégicas que han establecido con diferentes instancias (institucionales y académicas, entre otras). En el caso de UCKAM haber iniciado la comercialización en el mercado local y nacional, aunque aún no tienen clientes fijos. Para la Coordinadora de Productores de la Zona Centro del Estado de Veracruz, ganar premios por la calidad del café que producen (como la "taza de excelencia") ha sido el factor que les ha abierto las puertas de los mercados de especialidad. EI CORECAFECO considera que la calidad de su café ha permitido su amplia comercialización. En el caso de Ensambles de Cafés Mexicanos mencionó la buena calidad del café, las relaciones públicas, los contactos con otras instancias y el conocimiento del mercado. En VIDA consideran que contar con el apoyo de investigadores durante todo el proceso ha sido fundamental, así como el conocimiento de la calidad que demandan los clientes y la transparencia y trazabilidad en el proceso de producción y comercialización. Los Productores Sustentables de Ocozaca y la Unión Regional de Pequeños Productores de Café de la Región de Huatusco también coinciden en que la buena calidad del grano y contar con un precio base favorecen la comercialización.

Entre los principales problemas que han tenido las organizaciones y empresas para vender su café en los mercados diferenciados, Catuai Amarillo mencionó la falta de suficiente volumen de café por la afectación de la roya, la alta competencia que existe en los mercados de especialidad porque son nichos pequeños, la exigencia de mejora constante en la calidad del café en grano y taza, así como la deficiente mercadotecnia. Para la UCKAM y CORECAFECO el principal problema es la dificultad para encontrar nichos en el mercado donde se valore de forma justa el trabajo del productor, dado que 
existe mucha competencia y las ventanas de oportunidad no son amplias en dichos mercados. Por su parte la Coordinadora de Productores de la Zona Centro del Estado de Veracruz mencionó la reducida demanda de café certificado en comercio justo, los precios impuestos por la Bolsa de Nueva York, las desventajas en el tipo de cambio del peso mexicano con respecto al dólar y al euro, así como la incorporación de variedades más resistentes a enfermedades, pero con menor calidad del grano. Para Ensambles de Cafés Mexicanos, destacan los precios bajos en el mercado, la fuerte competencia en los mercados, los altos costos de envío y la falta de conocimiento del consumidor sobre el mayor costo que implica una buena calidad del café. VIDA y los Productores Sustentables de Ocozaca coincidieron en mencionar la insuficiencia del financiamiento del anticipo de acopio y la falta de infraestructura y tecnología para almacenar grandes cantidades de café sin que se demerite su calidad. Sólo la Unión Regional de Pequeños Productores de Café de la Región de Huatusco no mencionó ningún problema relevante que limite la comercialización en mercados diferenciados.

Finalmente, en cuanto a los retos para fortalecer la participación de los cafeticultores veracruzanos en los mercados diferenciados, Catuai Amarillo considera que es necesario incrementar la productividad de los cafetales, mejorar la fertilidad de los suelos, promover la diversificación productiva, mayor inversión en la modernización e innovación de la infraestructura y equipo de procesamiento de café, mejora continua de la calidad del producto en grano y taza, innovación en las estrategias de marketing (incluido un diseño atractivo del empaque), promoción y fortalecimiento del mercado nacional, regional y local. Otros retos de tipo social son la edad avanzada de los socios y la necesidad del relevo generacional, así como promover la equidad de género.

Por su parte, la UCKAM expresó que es importante fortalecer las organizaciones locales, potencializar el mercado nacional, mayor y más eficiente coordinación con las dependencias de gobierno directamente relacionadas con el sector cafetalero para implementar estrategias de mejora productiva de las fincas que redunden en incrementar la calidad del producto.

La Coordinadora de Productores de la Zona Centro del Estado de Veracruz añade que deben apoyarse y promoverse la variedad Typica como nicho de especialidad, para que la cafeticultura regional no dependa del tipo de cambio y la bolsa de valores; así mismo, apostar menos a las exportaciones y más a promover el mercado interno, mediante la educación del consumidor nacional en cuanto a los beneficios de comprar café de calidad.

El CORECAFECO indica que es necesario mejorar la trazabilidad del café, así como la creación de una instancia comercializadora a nivel nacional y un nuevo Instituto del Café que oriente las políticas públicas del sector cafetalero mexicano.

Ensambles de Cafés Mexicanos señala que se debe incentivar la productividad en las fincas y para esto se requiere que los productores tengan acceso a inversión y acompañamiento técnico; otros retos son fomentar la organización y alianzas entre pequeños productores; incentivar la educación del consumidor para que entiendan, diferencien y valoren el café de calidad; contrarrestar los precios bajos del café ya que esto deriva en un deterioro del manejo agronómico de las fincas y por tanto en un relajamiento de los procesos 
y controles de calidad del café; disminuir la dependencia de los precios internacionales y del acopio de las grandes empresas, ya que esto genera volatilidad de precios, inestabilidad y especulación.

Para VIDA resulta importante incentivar el consumo interno (nacional); promover que los productores no vendan su café en cereza, sino que logren procesarlo hasta pergamino para obtener mejores precios; incrementar la inversión en equipamiento para el beneficiado y capacitación técnica.

Los Productores Sustentables de Ocozaca visualizan como uno de los principales retos mantener $y$ cuidar la calidad de las variedades nativas y no introducir variedades desconocidas, ya que traen consigo plagas y enfermedades. Únicamente la Unión Regional de Pequeños Productores de Café de la Región de Huatusco, no emitió ninguna opinión respecto a este rubro.

\section{CONCLUSIONES}

Aunque existen diferencias en las opiniones expresadas por las organizaciones $y$ empresas estudiadas, en general los principales problemas que se mencionan para comercializar café en mercados diferenciados son: reducidos nichos en los mercados de especialidad, deficiencias en las estrategias de mercadotecnia, altos costos de exportación (incluidos los costos de certificación) y bajo consumo nacional. Mientras que los retos a superar para fortalecer la participación de los cafeticultores en los mercados diferenciados son: incrementar la productividad y calidad del café veracruzano, mejorar las estrategias de mercadotecnia y fomentar el consumo nacional mediante la educación del consumidor para que entiendan, diferencien y valoren el café de calidad.
La participación de las organizaciones y empresas de la región central de Veracruz en los mercados diferenciados de café, aunque es incipiente, representa un nicho de mercado con alto potencial como ventana de oportunidad para enfrentar la crisis productiva, siempre y cuando sea parte de una estrategia integral donde participen todos los actores de la cadena productiva de café.

\section{REFERENCIAS}

Batista, I. S. (20I8). Enfermedades del cultivo del café. República Dominicana: Unión Europea, Universidad ISA e Instituto Interamericano de Cooperación para la Agricultura.

Botey, J. (1990). Aproximación teórico-empírica a la figura del director de cuentas como gestor de la comunicación de marcar en agencias de publicidad de Barcelona (tesis de doctorado). Universitat Ramon Llull. Barcelona, España.

Castillo, M. (2019). La tradición del café de Veracruz. https://www.eluniversal.com.mx/menu/la-rutadel-cafe-veracruzano

Centro de Estudios para el Desarrollo Rural Sustentable y la Soberanía Alimentaria. (20I8). El café en México diagnóstico y perspectiva. México: Cámara de Diputados y CEDRSSA.

Ditsmarketing. (20I6). La marca paraguas. https://ditsmarketing.com/branding/la-marcaparaguas.html

Hermida, C. H. (20I8). Café de Veracruz, el de mayor calidad en el país.

https://www.uv.mx/prensa/reportaje/cafe-deveracruz-el-de-mayor-calidad-en-el-pais/

Hernández-Martínez, G. y Córdova, S. (20II). México, café y productores. Historia de la cultura cafetalera que transformó nuestras regiones. México: Centro Agroecológico del Café A.C.

Manson, R. M. (20I3). ¿Es posible imaginar un Veracruz sin café? http://www.inecol.edu.mx/inecol/index.php/es/20 13-06-05-10-34-10/17-ciencia-hoy/305-esposible-imaginar-un-veracruz-sin-cafe 
Manson, R.H., Contreras, A. y López-Barrera, F. (2008). Estudios de la biodiversidad en cafetales. En R. H. Manson, V. Hernández-Ortiz, S. Gallina y K. Mehltreter (Eds.), Agrosistemas cafetaleros de Veracruz. Biodiversidad, manejo y conservación (pp. I-I4). México: Instituto de Ecología A.C., e Instituto Nacional de Ecología.

Nava-Tablada, M. E. (20I2). Migración internacional y cafeticultura en Veracruz, México. Migraciones Internacionales. 6(3), |39-I7I.

Nava-Tablada, M. E. (2016). Mercados alternativos de café en el centro de Veracruz. México: El Colegio de Veracruz y Juan Pablos Editor.

Sosa Fernández, V. (2017). Los cafetales bajo sombra brindan mucho más que café.

https://www.inecol.mx/inecol/index.php/es/transp arencia-inecol//7-ciencia-hoy// 059-los-cafetalesbajo-sombra-brindan-mucho-mas-que-cafe

Williams-Linera, G. (20I5). El bosque mesófilo de montaña, veinte años de investigación ecológica ¿qué hemos hecho y hacia dónde vamos? Madera y Bosque. 2I, 5I-6I. 